\title{
QUALIDADE DE VIDA E ATIVIDADE FÍSICA: UM ESTUDO CORRELACIONAL EM IDOSOS COM HIPERTENSÃO ARTERIAL SISTÊMICA
}

\author{
Mayara Letícia Rinaldo \\ Patricia Carolina Borsato Passos ${ }^{2}$ \\ Francielli Ferreira da Rocha ${ }^{3}$ \\ Jéssica Luzia Milani ${ }^{3}$ \\ Lenamar Fiorese Vieira ${ }^{4}$
}

RINALDO, M. L.; PASSOS, P. C. B.; ROCHA, F. F. da; MILANI, J. L.; VIEIRA, L. F. Qualidade de vida e atividade física: um estudo correlacional em idosos com hipertensão arterial sistêmica. Arq. Cienc. Saúde UNIPAR, Umuarama, v. 20, n. 1, p, 51-57, jan./abr. 2016.

\begin{abstract}
RESUMO: O objetivo deste estudo foi investigar a relação entre qualidade de vida e o nível de atividade física de idosos com hipertensão arterial sistêmica da cidade de Mandaguaçu - PR. A amostra contou com 48 idosos de ambos os sexos com hipertensão arterial sistêmica, participantes do programa HIPERDIA. Como instrumentos foram utilizados o SF - 36, para analisar a qualidade de vida, e o IPAQ, para a mensuração do nível de atividade física. Para análise dos dados foram utilizados os testes "U" de Mann-Whitney, Kruskal Wallis e o Spearman $(\mathrm{p} \leq 0,05)$. Os idosos que obtiveram níveis altos de atividade física $(83,3 \%)$ foram considerados ativos. Houve correlações significativas entre a qualidade de vida nas dimensões capacidade funcional, limitação por aspectos físicos e vitalidade com atividade física moderada e vigorosa. Ao final da pesquisa, foi possível concluir que quanto mais os idosos realizam atividades físicas moderadas e vigorosas, melhor é a qualidade de vida no aspecto da capacidade funcional.
\end{abstract}

PALAVRAS-CHAVE: Atividade Física. Idosos. Qualidade de vida.

\section{QUALITY OF LIFE AND PHYSICAL ACTIVITY: A CORRELATIONAL STUDY IN ELDERLY WITH SYSTEMIC HYPERTENSION}

\begin{abstract}
The aim of this study was to investigate the relationship between the quality of life and the level of physical activity of elderly people with Systemic Arterial Hypertension in the city of Mandaguaçu - PR. The sample had 48 elderly people from both genders with hypertension. These people participated in the HIPERDIA program. The SF - 36 instrument was used to analyze the quality of life, and IPAQ for measuring the level of physical activity. For data analysis, the "U" Mann-Whitney, Kruskal Wallis and Spearman tests were used $(\mathrm{p} \leq 0.05)$. The elderly who had high levels of physical activity $(83.3 \%)$ were considered active. There were significant correlations between the quality of life in functional capacity, limitations on physical aspects and vitality aspects with moderate and vigorous physical activity. At the end of the research, it was possible to conclude that the more the participants practiced moderate and vigorous physical activities, the better it was for the quality of life in the functional capacity aspect.
\end{abstract}

KEYWORDS: Elderly. Quality of life. Physical activity.

\section{Introdução}

A Organização Mundial da Saúde aponta que nas últimas décadas ocorreu um aumento no número de pessoas idosas tanto em países desenvolvidos como em desenvolvimento (WORLD HEALTH ORGANIZATION, 2007). Atualmente no Brasil, estima-se que para o ano de 2050 a expectativa de vida seja de 81,3 anos e, ainda, que o país será o sexto do mundo considerando o número da população idosa (IBGE, 2010). Como consequência, espera-se um aumento de casos de doenças que estejam relacionadas ao envelhecimento (MELO et al., 2014).

Nascimento (2007) aponta que, com o tempo, o organismo humano sofre um processo natural de envelhecimento, passando assim por diversas transformações funcionais e estruturais, diminuindo a vitalidade e abrindo portas para possíveis doenças crônicas, afetando a qualidade de vida das pessoas.

Dentre as doenças crônicas não transmissíveis encontra-se a hipertensão arterial sistêmica, que acomete $65 \%$ dos idosos brasileiros e é considerada um dos fatores de risco que mais contribui para o surgimento de doenças cardiovasculares, renais e cerebrovasculares. A hipertensão é responsável por $25 \%$ das mortes por doença arterial coronariana, $40 \%$ das mortes relacionadas com acidente vascular cerebral e $50 \%$ das mortes relacionadas com insuficiência renal terminal (MINISTÉRIO DA SAÚDE CADERNO DE ALTERAÇÕES BÁSICAS, 2006).

Diante dos dados apresentados, percebe-se a importância de investigar aspectos que possam ser intervenientes na vida de idosos com hipertensão arterial. Maciel (2010) aponta que a prática constante de atividade física pode melhorar a qualidade de vida e ajudar na prevenção de doenças coronarianas, entre as quais se destaca a hipertensão arterial.

Estudos encontrados na literatura brasileira referentes à atividade física com idosos têm buscado avaliar a atividade física e o estado de saúde mental de idosos (BENEDETTI et al., 2008), assim como a prevenção e o tratamento de diferentes doenças (COELHO; BURINI, 2009; FIGUEIREDO NETO; QUELUZ; FREIRE, 2011; SILVA, NAHAS, 2004). Contudo, a literatura apresenta uma lacuna no que diz respeito a estudos que investiguem qualidade de vida e ativi-

DOI: https://doi.org/10.25110/arqsaude.v20i1.2016.5472

${ }^{1}$ Graduada em Educação Física pela Universidade Estadual de Maringá.

${ }^{2}$ Professora Mestre em Educação Física na Universidade Paranaense e Prefeitura de Maringá. Discente em nível de doutorado no Programa de Pós-Graduação Associado em Educação Física UEM/UEL.

${ }^{3}$ Universidade Estadual de Maringá.

${ }^{4}$ Professora Doutora no Departamento de Educação Física na Universidade Estadual de Maringá. Docente no Programa de Pós-Graduação Associado em Educação Física UEM/UEL. 1fvieira@uem.br 
dade física, considerando a população de idosos hipertensos.

Mas também, a qualidade de vida tem sido estudada em diferentes tipos de populações (KUNKEL; OLIVEIRA; PERES, 2009; MORAES et al., 2012), diversas condições clínicas (STEIN et al., 2012; CARVALHO et al., 2013) e utilizando uma grande variedade de tipos de instrumentos de pesquisa (CICONELLI et al., 1997; FLECK et al., 1999; ALVES, 2011; KAWAKAME; MIYADAHIRA, 2005; ALVES, 2011).

No geral, a qualidade de vida abrange aspectos que se relacionam à saúde, tais como o bem-estar físico, funcional, emocional e mental, incluindo ainda fatores de relacionamento com os amigos, a família, o trabalho, entre outros (MAZO, 2008). Neste sentido, ressalta-se a importância de como o indivíduo idoso e com hipertensão percebe a qualidade de vida.

Na busca por aspectos que possam contribuir para a população de idosos hipertensos, é indispensável considerar a atividade física e sua relação com a qualidade de vida, na perspectiva do idoso (TAKANO et al., 2010). Percebe-se a escassez de os estudos com idosos hipertensos, no contexto nacional, que investiguem esta relação, devido à expectativa do crescimento desta população e sua importância (CARVALHO et al., 2013), em especial o impacto na saúde pública. Face às considerações, este estudo teve como objetivo investigar a relação entre qualidade de vida e o nível de atividade física de idosos hipertensos da cidade de Mandaguaçu - PR.

\section{Métodos}

O estudo de caráter descritivo correlacional foi realizado com idosos que apresentavam hipertensão arterial sistêmica, participantes do programa HIPERDIA (Sistema de Gestão Clínica de Hipertensão Arterial e Diabetes Mellitus da Atenção Básica), cadastrados no Centro de Saúde de Mandaguaçu - PR.

A amostra foi composta por 48 idosos, sendo 21 do sexo masculino e 27 do sexo feminino, com 65 anos ou mais. Como critério de inclusão, os participantes deveriam ter sido diagnosticados hipertensos há mais de seis meses, estarem utilizando de forma correta, conforme prescrição médica, a medicação anti-hipertensiva e entregarem o Termo de Consentimento Livre e Esclarecido (TCLE), devidamente assinado. Foram excluídos deste estudo os sujeitos com idade inferior a 65 anos e diagnosticados com diabetes mellitus.

Como local o Centro de Saúde foi escolhida a Unidade Básica de Saúde de Mandaguaçu, por ter a estrutura mais completa da cidade e também por incluir mais hipertensos cadastrado sem relação às outras Unidades de Saúde da mesma cidade.

Para avaliar a Qualidade de vida foi utilizado o questionário Medical Outcomes Study 36 - Item Short-Form Health Survey (SF-36), validado por Ciconelli (1997). O SF36 é um instrumento genérico de avaliação da qualidade de vida, simples de compreender e de ser administrado. É um questionário multidimensional composto por 36 itens, englobando 8 escalas ou componentes: capacidade funcional, aspectos físicos, dor, estado geral da saúde, vitalidade, aspectos sociais, aspectos emocionais e saúde mental. Proporciona um escore final de 0 a 100, no qual zero vai corresponder ao pior estado geral de saúde e 100 ao melhor estado de saúde.
Para avaliar a atividade física foi utilizada a versão curta do International Physical Activity Questionnaire (IPAQ), desenvolvido por Craig et al. (2003). Esse questionário é composto por 7 questões que permitem estimar o tempo gasto em atividades físicas moderadas ou vigorosas na semana, em diversas situações diárias tais como: trabalho, transporte, tarefas domésticas e lazer, e ainda o tempo gasto em atividades mais tranquilas, que são realizadas sentadas. Para a avaliação dos dados da atividade física foi utilizado o acordo realizado entre o Centro Estudos Laboratório de Aptidão Física São Caetano Do Sul CELAFISCS e o Center for Disease Control (CDC) de Atlanta em 2002, referido no estudo de Matsudo et al. (2001).Esse instrumento foi desenvolvido para indivíduos adultos, mas, devido à ausência de um instrumento específico para a população de idosos no projeto inicialmente submetido e aprovado pelo comitê de ética, e buscando suprir a dificuldade dos indivíduos em desprender tempo para participar do estudo, optou-se por utilizar sua versão curta,na qual os pesquisadores realizaram um estudo piloto para treinamento da coleta de dados. Os critérios de frequência e duração assim como a classificação ocorrem em quatro categorias:

1- Muito ativo (realiza atividade vigorosa: $\geq 5$ dias/ sem e $\geq 30$ minutos por sessão e/ou vigorosa: $\geq 3$ dias/sem e $\geq 20$ minutos por sessão + moderada e/ ou caminhada: $\geq 5$ dias/sem e $\geq 30$ minutos por sessão);

2- Ativo (atividade vigorosa: $\geq 3$ dias/sem e $\geq 20 \mathrm{mi-}$ nutos por sessão; e/ou moderada ou caminhada: $\geq 5$ dias/sem e $\geq 30$ minutos por sessão; e/ou qualquer atividade somada: $\geq 5$ dias/sem e $\geq 150$ minutos/ sem;

3- Irregularmente ativo: aquele que realiza atividade física, porém insuficiente para ser classificado como ativo, pois não cumpre as recomendações quanto à frequência ou duração. Para alcançar essa classificação, soma-se a frequência e a duração dos diferentes tipos de atividades;

4- Sedentário: aquele que não realizou nenhuma atividade física por pelo menos 10 minutos contínuos durante a semana.

Contudo, este estudo utilizou somente as dimensões muito ativo, ativo, irregularmente ativo e sedentário, pois nenhum idoso foi classificado no irregularmente ativo B.

A coleta dos dados ocorreu durante o ano de 2013 e teve a duração de três meses (abril, junho e agosto). A aplicação dos instrumentos foi realizada durante as reuniões marcadas pelo programa HIPERDIA, que ocorrem de dois em dois meses, e também a partir de agendamento na casa dos sujeitos. Os instrumentos foram respondidos individualmente e/ou com auxílio dos pesquisadores, pois vários idosos não eram alfabetizados, a coleta teve a duração de, aproximadamente, 25 minutos.

Esta pesquisa está vinculada ao projeto institucional "Impacto de variáveis psicológicos sobre comportamentos relacionados à saúde", com parecer aprovado do comitê de ética $n^{\circ}$ 238/2011, na Universidade Estadual de Maringá. Este projeto teve a duração de quatro anos, cujo o principal objetivo foi investigar o impacto das variáveis psicológicas sobre comportamentos relacionados à saúde, os participantes do projeto foram acadêmicos de graduação, pós graduação, 
professores/pesquisadores e coordenado por doutores desta instituição.

\section{Análise Estatística}

Para análise dos dados foram utilizadas estatística descritiva e inferencial. Para verificação da normalidade, utilizou-se o teste Shapiro Wilk, a partir do qual foi observada sua não normalidade. Para a apresentação dos resultados, utilizou-se a Mediana (Md), como medida de tendência central, e Quartis (Q1; Q3), como separatrizes. O Teste "U" de Mann-Whitney foi utilizado para a comparação entre os sexos; para a comparação entre as faixas etárias foi utilizado o Kruskal Wallis, quando encontrada diferença, aplicou-se "U” de Mann-Whitney. Para a correlação foi utilizado o teste de Spearman, o nível de significância adotado foi de $\mathrm{p} \leq 0,05$ e o pacote estatístico foi oStatistical Package for the Social Sciences (SPSS) 13.0.

\section{Resultados e Discussões}

Os resultados evidenciaram que a média da idade foi de $72,77( \pm 5,24)$ anos, sendo $43,75 \%(n=21)$ eram do sexo masculino e $56,25 \%(n=27)$ eram do sexo feminino. Os idosos apresentaram escores altos nos domínios de qualidade de vida (Tabela 1), na "capacidade funcional" $(\mathrm{Md}=75,00)$, seguido de "aspectos emocionais" $(\mathrm{Md}=66,70)$. Os menores escores da qualidade de vida foram nos domínios o domínio "dor" $(\mathrm{Md}=30,00)$ e "estado geral de saúde" $(\mathrm{Md}=25,00)$.

Tabela 1: Dados descritivos dos escores da qualidade de vida de idosos ( $n=48)$ com hipertensão arterial sistêmica de Mandaguaçu.

\begin{tabular}{lcc}
\hline Qualidade de Vida & Md & Q1-Q3 \\
\hline Capacidade Funcional & 75,0 & $(62,5-090,0)$ \\
Aspectos Emocionais & 66,7 & $(33,3-100,0)$ \\
Limitação por Aspectos Físicos & 62,5 & $(50,0-100,0)$ \\
Saúde Mental & 56,0 & $(52,0-064,0)$ \\
Vitalidade & 52,5 & $(45,0-060,0)$ \\
Aspectos Sociais & 50,0 & $(31,3-050,0)$ \\
Dor & 30,0 & $(00,0-040,0)$ \\
Estado Geral de Saúde & 25,0 & $(20,0-032,5)$ \\
\hline
\end{tabular}

Brito et al. (2008) encontraram resultados semelhantes, demonstrando que os aspectos da qualidade de vida com os menores escores foram a "dor" e o "estado geral de saúde". O domínio "estado geral de saúde" pode ter relações com as manifestações associadas à hipertensão arterial e ao seu tratamento. Os autores apontam que a hipertensão arterial é considerada silenciosa e assintomática, porém, estudos realizados demonstraram que portadores da doença se referiram a alguns sintomas como dores de cabeça, dor no peito, alterações dos batimentos cardíacos e outros.

Toscano e Oliveira (2009) encontraram resultados consoantes relacionados aos "aspectos físicos" e "emocionais", porém, um dos menores escores foi para a "capacidade funcional". Figueiredo Neto et al. (2011), avaliaram a qualidade de vida e a sua relação com a atividade física nas distintas situações diárias, em pacientes acima de 40 anos, utilizando o SF-36, encontrando também um dos maiores escores para "aspectos emocionais", porém, o menor escore ocorreu para "aspectos físicos".

Ao contrário dos resultados deste estudo, Magalhães e Iório (2011) apontam o menor escore no "aspecto físico", o qual tem relação direta com a capacidade do indivíduo de realizar determinadas atividades físicas, como, por exemplo, caminhar, subir degraus de escada e fazer as atividades domésticas.

A Tabela 2 demonstra que a maioria foi considerada como "ativos" $(83,3 \%)$, corroborando o estudo de Benedetti et al (2008), no qual foi constatado que a maioria dos idosos de Florianópolis foi considerada ativa. Em concordância, Silva et al. (2012) realizaram uma pesquisa com idosos utilizando o IPAQ e verificaram que a maioria dos entrevistados também era de "ativos". Assim como outros estudos sobre a mesma perspectiva, percebe-se que a atividade física pode ser considerada um fator essencial para a manutenção da boa saúde e prevenção de doenças crônicas em idosos (DOMINGUES; NERI, 2009).

Tabela 2: Frequência do nível de atividade física de idosos com hipertensão arterial sistêmica de Mandaguaçu

\begin{tabular}{lcc}
\hline Atividade física & F(n) & Percentual (\%) \\
\hline Muito Ativo & 04 & 08,3 \\
Ativo & 40 & 83,3 \\
Irregularmente Ativo & 03 & 06,3 \\
Sedentário & 01 & 02,1 \\
Total & 48 & 100,0 \\
\hline
\end{tabular}

No entanto, no estado de Pernambuco foram encontrados níveis altos de sedentarismo $(68,3 \%)$ entre idosos (ALVES et al., 2010). Os autores concluíram que o sedentarismo deve ser combatido, pois é um fator de risco importante para as doenças crônicas não transmissíveis. Sendo assim, Matsudo (2002) destaca que a atividade física é indispensável para a qualidade de vida durante o envelhecimento, prevenindo e controlando doenças crônicas não transmissíveis que são mais frequentes na terceira idade, como as doenças cardiovasculares e o câncer.

A Tabela 3 demonstra que o sexo feminino apresentou maior mediana na duração da atividade física moderada $(p=0,002)$ em relação ao sexo masculino, indicando que as mulheres idosas têm maior prática de atividade física moderada do que os homens; os demais aspectos não apresentaram diferença estatística. Ressalta-se que os valores do IPAQ representam o tempo de atividade em minutos, por semana. 
Tabela 3: Comparação da Qualidade de Vida e Atividade Física dos idosos com hipertensão arterial sistêmica, em função do sexo.

\begin{tabular}{lccc}
\hline \multirow{2}{*}{ DIMENSÕES } & Masculino (21) & Feminino (27) & P \\
\cline { 2 - 3 } SF-36 & Md (Q1 - Q3) & Md (Q1 - Q3) & 0,601 \\
Capacidade Funcional & $75,0(70,0-85,0)$ & $80,0(60,0-90,0)$ & 0,545 \\
Limitação por Aspectos Físicos & $50,0(50,0-100,0)$ & $75,0(37,5-100,0)$ & 0,073 \\
Dor & $20,0(00,0-30,0)$ & $30,0(10,0-50,0)$ & 0,559 \\
Estado Geral de Saúde & $25,0(20,0-30,0)$ & $25,0(20,0-32,5)$ & 0,100 \\
Vitalidade & $60,0(50,0-60,0)$ & $50,0(45,0-55,0)$ & 0,513 \\
Aspectos Sociais & $50,0(25,0-50,0)$ & $50,0(43,8-50,0)$ & 0,284 \\
Aspectos Emocionais & $100,0(33,3-100,0)$ & $66,7(0,0-100,0)$ & 0,219 \\
Saúde Mental & $60,0(52,0-64,0)$ & $56,0(50,0-60,0)$ & 0,229 \\
IPAQ & & & $0,002^{* *}$ \\
Caminhada & $40,0(20,0-60,0)$ & $30,0(12,5-57,5)$ & 0,185 \\
Moderada & $15,0(10,0-30,0)$ & $60,0(30,0-165,0)$ & $00,0(0,0-25,0)$ \\
Vigorosa & $00,0(00,0-00,0)$ & & \\
\hline Difaç & \\
\hline
\end{tabular}

Diferença estatisticamente significativa ${ }^{*} \mathrm{p} \leq 0,05 .{ }^{* *} \mathrm{p} \leq 0,01$.

Carvalho et al. (2011) encontraram resultados semelhantes ao pesquisarem o nível de atividade física de servidores idosos em período de pré-aposentadoria da Universidade Federal do Maranhão (UFMA), percebendo que as mulheres são mais ativas que os homens. Em contrapartida, uma pesquisa de Benedetti et al. (2007) mostra que os homens idosos foram considerados mais ativos do que as mulheres. O estudo de Tribess, Virtuoso Junior e Oliveira (2012), realizado com indivíduos de idade semelhante aos desta pesquisa, não indicou diferenças significativas na comparação da prática de atividade física moderada em função do sexo.

A Tabela 4 mostra, na dimensão "aspecto social", diferenças estatísticas $(p=0,035)$ ao considerar a qualidade de vida. Na comparação do nível de atividade física entre as faixas etárias dos idosos, houve diferença significativa para "atividade física vigorosa" $(\mathrm{p}=0,008)$. Esses resultados demonstraram que quanto mais jovem o idoso, melhor este considera sua qualidade de vida nos aspectos sociais.

Tabela 4: Comparação da Qualidade de Vida e o nível de Atividade Física em função da faixa etária de idosos com hipertensão arterial sistêmica de Mandaguaçu.

\begin{tabular}{lcccc}
\hline \multicolumn{1}{c}{ Dimensões } & 65 a 70 anos $(\mathrm{n}=20$ & 71 a 75 anos $(\mathrm{n}=11)$ & 76 ou mais anos $(\mathrm{n}=17)$ \\
\cline { 2 - 4 } & Md $(\mathrm{Q} 1-\mathrm{Q} 3)$ & Md $(\mathrm{Q} 1-\mathrm{Q} 3)$ & Md $(\mathrm{Q} 1-\mathrm{Q} 3)$ & \\
\hline SF-36 & & & & \\
Capacidade Funcional & $87,5(65,0-90,0)$ & $75,0(70,00-90,00)$ & $70,0(60,0-80,0)$ & 0,203 \\
Limitação por Aspectos Físicos & $75,0(2,5-100,0)$ & $75,0(37,5-100,0)$ & $50,0(50,0-100,0)$ & 0,926 \\
Dor & $30,0(00,0-50,0)$ & $30,0(20,0-35,0)$ & $20,0(00,0-40,0)$ & 0,859 \\
Estado Geral de Saúde & $25,0(20,0-30,0)$ & $30,0(20,0-40,0)$ & $25,0(20,0-40,0)$ & 0,577 \\
Vitalidade & $52,5(45,0-60,0)$ & $50,0(47,5-57,5)$ & $60,0(40,0-70,0)$ & 0,583 \\
Aspectos Sociais & $50,0(50,0-62,5)^{\mathrm{ab}}$ & $50,0(50,0-75,0)^{\mathrm{ac}}$ & $50,0(25,0-50,0)^{\mathrm{bc}}$ & $0,014 * *$ \\
Aspectos Emocionais & $66,7(00,0-100,0)$ & $33,3(16,7-100,0)$ & $100,0(66,7-100,0)$ & 0,185 \\
Saúde Mental & $60,0(52,0-64,0)$ & $56,0(54,0-58,0)$ & $52,0(48,0-64,0)$ & 0,724 \\
IPAQ & & & & 0,527 \\
Caminhada & $30,0(20,0-62,5)$ & $40,0(30,0-60,0)$ & $30,0(10,0-60,0)$ & 0,527 \\
Moderada & $45,0(30,0-105,0)$ & $60,0(20,0-90,0)$ & $15,0(10,0-120,0)$ & 0,350 \\
Vigorosa & $15,0(00,0-35,0)^{\mathrm{d}}$ & $00,0(00,0-00,0)^{\mathrm{e}}$ & $00,0(00,0-00,0)^{\mathrm{de}}$ & $0,008^{* *}$ \\
\hline
\end{tabular}

Diferença estatisticamente significativa ${ }^{*} \mathrm{p} \leq 0,05 .{ }^{* *} \mathrm{p} \leq 0,01$.

Aspecto Social: $\mathrm{a}=65$ a 70 anos com 71 a 75 anos ( $\mathrm{p}=0,035) ; \mathrm{b}=65$ a 70 anos com 76 anos ( $=0,010)$; $\mathrm{c}=71$ a 75 anos com 76 anos ou mais $(\mathrm{p}=0,018)$. Atividade física vigorosa: $\mathrm{d}=65$ a 70 anos com 76 anos ou mais $(\mathrm{p}=0,010)$; e 71 a 75 anos com 76 anos ou mais $(\mathrm{p}=0,018)$. 
Um estudo de Brito et al. (2008) analisou o fator dos "aspectos sociais", evidenciando a integração das pessoas em atividades sociais. Para os portadores de hipertensão arterial, participar de grupos é um grande desafio, pois a doença aumenta a dificuldade de manter relações sociais, levando o idoso a comprometer sua percepção de qualidade de vida. Em estudo de Magalhães e Iório (2011) também foram obtidos resultados significantes em relação à escala social em função do sexo e da faixa etária dos idosos.

A atividade física vigorosa (Tabela 4), quando comparada entre idosos de 65 a 70 anos e idosos de 76 anos ou mais, indicou diferença estatisticamente significativa ( $\mathrm{p}=$ 0,01). Na comparação entre a faixa etária de 71 a 75 anos e a de 76 anos ou mais também houve diferença estatística ( $p=$ $0,02)$, porém, ao comparar idosos de 65 a 70 anos com os de 71 a 75 anos, nenhuma diferença estatística foi encontrada, demonstrando que quanto mais jovem o idoso, mais intenso a prática de atividade física. Os valores da mediana são referentes ao cálculo do tempo de atividade em minutos e por semana.

Em concordância com o presente estudo, Castro et al. (2010) utilizaram o IPAQ e observaram que os idosos mais jovens praticam mais atividades físicas que os idosos mais velhos. Domingos e Neri (2009) observaram que os idosos mais jovens tiveram uma maior frequência de atividade física $(50,8 \%)$ quando comparados aos mais velhos $(17,6 \%)$. Tri- bess, et al. (2009) destaca que com o tempo (envelhecimento), os indivíduos diminuem a sua participação nas práticas de atividades físicas.

$\mathrm{Na}$ Tabela 5, a correlação positiva foi significativa entre a "atividade física moderada" e a "capacidade funcional" ( $\mathrm{r}=0,29)$, assim como entre "atividade física vigorosa" e "capacidade funcional" ( $r=0,37)$, levando à conclusão de que quanto mais os idosos praticam atividades moderadas e vigorosas, melhor é a capacidade funcional. Porém, apesar dos resultados serem significativos, os valores de correlação até 0,39 são considerados fracos, somente os valores de correlação entre 0,40 e 0,59 podem ser considerados moderados (MITRA; LANKFORD, 1999). É importante considerar as especificidades dos diferentes instrumentos de pesquisa, pois é comum entre estudiosos da área da psicologia do esporte utilizando questionários psicométricos subjetivos, a obtenção de resultados de correlação a partir de 0,30; quando são significativos, os dados tornam-se relevantes.

Houve correlação moderada e significativa entre a "atividade física moderada" com "limitações por aspectos físicos" $(\mathrm{r}=0,52)$ e "atividade física vigorosa" com "limitações por aspectos físicos" $(\mathrm{r}=0,36)$, demonstrando que quanto mais atividade física moderada e vigorosa praticam, melhor os idosos se percebem na dimensão "limitações por aspectos físicos".

Tabela 5: Coeficiente de correlação da Qualidade de Vida com o nível de Atividade Física de idosos com hipertensão arterial sistêmica de Mandaguaçu.

\begin{tabular}{|c|c|c|c|c|c|}
\hline $\mathrm{SF}-36 \quad$ IPAQ & Caminhada & Moderada & Vigorosa & $\begin{array}{l}\text { Sentado Durante a } \\
\text { Semana }\end{array}$ & $\begin{array}{c}\text { Sentado Durante o Final } \\
\text { de Semana }\end{array}$ \\
\hline Capacidade Funcional & 0,191 & $0,29 *$ & $0,37 * *$ & $-0,18$ & $-0,19$ \\
\hline Limitação por Aspectos Físicos & 0,18 & $0,52 * *$ & $0,36^{*}$ & 0,11 & 0,10 \\
\hline Dor & $-0,10$ & $-0,06$ & $-0,13$ & $-0,17$ & 0,00 \\
\hline Estado Geral de Saúde & $-0,22$ & 0,03 & $-0,22$ & $-0,15$ & $-0,23$ \\
\hline Vitalidade & $-0,28$ & $-0,15$ & $-0,36^{*}$ & $0,37 * *$ & 0,24 \\
\hline Aspectos Sociais & $-0,19$ & $-0,14$ & $-0,02$ & $-0,09$ & $-0,16$ \\
\hline Aspectos Emocionais & 0,15 & 0,12 & 0,24 & $-0,34$ & 0,10 \\
\hline Saúde Mental & 0,09 & 0,07 & 0,01 & 0,00 & 0,10 \\
\hline
\end{tabular}

Vitorino et al. (2012) perceberam que os idosos que realizavam atividades físicas foram relacionados aos domínios físicos e psicológicos. Esses achados vão ao encontro da revisão sistemática de Oliveira et al. (2010), que pesquisaram o impacto da atividade física na qualidade de vida de idosos, percebendo que a frequência com que os idosos praticam atividades físicas influencia a melhoria da qualidade de vida, ajudando na realização de atividades diárias, mantendo a energia e vigor, diminuindo as incapacidades motoras e ajudando o idoso a manter a sua autonomia.

Brito et al. (2008) avaliaram a qualidade de vida de portadores de hipertensão arterial e verificaram que as limitações por aspectos físicos dizem respeito ao tipo e quantidade de trabalho; quando tais limitações impedem o indivíduo de realizar as atividades diárias, os autores perceberam que os pacientes não demostraram incapacidades físicas para desenvolverem suas atividades habituais.

Outra correlação significativa foi entre a "atividade física vigorosa" e "vitalidade" $(\mathrm{r}=-0,36)$. Percebeu-se que quanto mais atividades vigorosas são praticadas, mais baixa é a vitalidade dos idosos. Entre os aspectos "sentado durante a semana" e a "vitalidade" $(\mathrm{r}=0,37)$ foi indicado que quanto mais tempo os idosos permanecem sentados durante a semana, maior é sua percepção de vitalidade.

Silva et al. (2010) estabeleceram uma relação entre qualidade de vida e atividade física utilizando o instrumento WHOQOL, com questionário de atividades físicas habituais, e perceberam que os indivíduos muito ativos tiveram maiores escores de qualidade de vida quando comparados aos inativos.

\section{Conclusão}

A qualidade de vida em geral dos idosos diagnosticados com hipertensão arterial sistêmica foi percebida como boa, apresentando valores mais altos para os aspectos de ca- 
pacidade funcional, limitação por aspectos físicos e emocionais. Quanto ao nível de atividade física, percebeu-se que a maior parte dos idosos hipertensos foi considerada como ativos, ressalta-se que o sexo feminino pratica mais atividades físicas moderadas quando comparado ao sexo masculino. Ao analisar a qualidade de vida e o nível de atividade física em função da faixa etária, evidenciou-se que quanto mais jovem for o idoso melhores são as suas percepções a respeito das relações sociais.

Finalmente, a relação entre qualidade de vida e atividade física demonstrou que quanto mais os idosos realizam atividades físicas moderadas e vigorosas, melhor percebem a qualidade de vida no aspecto da capacidade funcional, que está relacionado à capacidade da realização de atividades simples do cotidiano, como tomar banho, vestir-se, caminhar alguns metros, alcançar e carregar mantimentos e outros.

A presente pesquisa teve algumas limitações, tais como o baixo interesse dos idoso sem responder os questionários, o que consequentemente levou a um $\mathrm{n}$ amostral baixo; caráter transversal do estudo, devido ao impedimento de estabelecimento de uma relação causa-efeito e a utilização do IPAQ, considerado um instrumento que em si já apresenta várias limitações: é confuso para alguns idosos e não é uma medida objetiva. Sugere-se que estudos futuros com a população de idosos utilizem a versão longa do IPAQ, validada para esta população, e que selecionem uma população maior, não somente de um local, finalmente, se possível, sugere-se que a pesquisa seja realizada com caráter longitudinal.

\section{Referências}

ALVES, E. F. Qualidade de vida: considerações sobre os indicadores e instrumentos de medida. Revista Brasileira de Qualidade de Vida, v. 3, n.1, p.16-23, 2011.

ALVES, J. G. B. et al. Prevalência de adultos e idosos insuficientemente ativos moradores em áreas de unidades básicas de saúde com e sem Programa Saúde da Família em Pernambuco, Brasil. Caderno de Saúde Pública, v. 26, n. 3, p. 543-556, 2010.

BENEDETTI, T. R. B. et al. Atividade física e estado de saúde mental de idosos. Revista de Saúde Pública, v. 42, n. 2, p. 302-307, 2008.

BENEDETTI, T. R. B. et al. Reprodutibilidade e validade do questionário internacional de atividade física (IPAQ) em homens idosos. Revista Brasileira de Medicina do Esporte, v.13, n.1, p.11-16, 2007.

BRASIL. Ministério da Saúde. Cadernos de Atenção Básica: hipertensão arterial sistêmica. n. 15, 2006. (Série Cadernos de Atenção Básica, Série A).

BRITO, D. M. S. et al. Qualidade de vida e percepção da doença entre portadores de hipertensão arterial. Caderno de Saúde Pública, v. 24, n. 4, p. 933-940, 2008.

CAMPOLINA, G.; DINI, P. S.; CICONELLI, R. M. Impacto da doença crônica na qualidade de vida de idosos da comunidade em São Paulo (SP, Brasil). Revista Ciência e Saúde Coletiva, v.16, n. 6, p. 2919-2925, 2011.

CARVALHO, C. A. et al. Nível de atividade física de servidores idosos em período de pré-aposentadoria da Universidade Federal do Maranhão - UFMA. Revista de Pesquisa em Saúde, v.12, n. 2, p. 32-37, 2011.

CARVALHO, M. V. et al. A influência da hipertensão arterial na qualidade de vida. Arquivos Brasileiros de Cardiologia, v.100, n. 2, p.164-174, 2013.

CASTRO, J. et al. Nível de atividades físicas em mulheres idosas frequentadoras das agências de assistência à saúde do idoso residentes em Belém do Pará. Revista Brasileira de Ciência e Movimento, v.18, n. 4, p. 39-44, 2010.

COELHO, C. F.; BURINI, R. C. Atividade física para prevenção e tratamento das doenças crônicas não transmissíveis e da incapacidade funcional. Revista de Nutrição, v. 22, n. 6, p. 937-946, 2009.

CRAIG, C. L. et al. International physical activity questionnaire: 12-country reliability and validity. Medicine and Science Sports and Exercise, v. 35, n. 8, p.1381-95, 2003.

DOMINGUES, P. C.; NERI, A. L. Atividade física, sintomas e doenças auto-relatadas em idosos da comunidade. Revista Brasileira de Atividade Física e Saúde, v.14, n. 3, p.164-173, 2009.

FLECK, M. P. A. et al. Desenvolvimento da versão em português do instrumento de avaliação de qualidade de vida da OMS (WHOQOL-100). Revista Brasileira de Psiquiatria, v. 21, n.1, p.19-28, 1999.

IBGE - INSTITUTO BRASILEIRO DE GEOGRAFIA E ESTATÍSTICA, 2010: Disponível em: http://www.ibge. gov.br/home/presidencia/noticias/noticia_visualiza.php?id_ noticia $=1866 \& i d \_p a g i n a=1$. Acesso em: 08/02/2013.

KAWAKAME, P. M. G.; MIYADAHIRA, A. M. K. Qualidade de vida de estudantes de graduação em enfermagem. Revista da Escola de Enfermagem USP, v. 39, n. 2, p.164-172, 2005.

KUNKEL, N.; OLIVEIRA, W. F.; PERES, M. A. Excesso de peso e qualidade de vida relacionada à saúde em adolescentes de Florianópolis, SC. Revista de Saúde Pública, v. 43, n. 2, p. 226-235, 2009.

MACIEL, M. G. Atividade física e funcionalidade do idoso. Revista Motriz, Rio Claro, v.16 n. 4, p.1024-1032, 2010.

MAGALHÃES, R.; IÓRIO, M. C. M. Qualidade de vida e restrição de participação: um estudo em idosos. Brazilian Journal of Otorhinolaryngology, v.77, n. 5, p. 628-638, 2011.

MATSUDO, S. M. Envelhecimento, atividade física e saúde. Revista Mineira de Educação Física, v.10, n.1, 
p.195-209, 2002.

MATSUDO, S. M. et al. Questionário internacional de atividade física (IPAQ): estudo de validade e reprodutibilidade no Brasil. Revista de Atividade Física e Saúde, v. 6, n. 2, p. 5-18, 2001.

MAZO, G. Z. Atividade física, qualidade de vida e envelhecimento. Porto Alegre: Sulina, 2008.

MELO, B. et al. Efeito do treinamento físico na qualidade de vida em idosos com depressão maior. Revista Brasileira de Atividade Física e Saúde, v.19, n. 2, p. 205-214, 2014.

MITRA, A.; LANKFORD, S. Research methods in park, recreation and leisure services. Champaign: Sagamore Publishing, 1999.

MORAES, K. et al. Efeitos de três programas de treinamento de força na qualidade de vida de idosas.

Revista Brasileira de Atividade Física e Saúde, v.17, n. 3, p.181-187, 2012.

NASCIMENTO, R. F. L. Depressão e o desempenho cognitivo em idosos através do teste Wisconsin classificação de cartas (WCST). 2007. 64 f. Dissertação (Mestrado em Psicologia) - PUCRS, Faculdade de Psicologia - Centro de Ciências da Saúde - Pontifícia Universidade Católica do Rio Grande do Sul, Porto Alegre, 2007.

NATIONAL HEALTH ACCOUNTS, World Health Organization, 2007.

FIGUEIREDO NETO, E. M.; QUELUZ, T. T.; FREIRE, B. F. A. Atividade física e sua associação com qualidade de vida em pacientes com osteoartrite. Revista Brasileira de Reumatologia, v. 51, n. 6, p. 539-549, 2011.

OLIVEIRA, A. C. et al. Qualidade de vida em idosos que praticam atividade física uma revisão sistemática. Revista Brasileira de Geriatria e Gerontologia, v.13, n. 2, p. 301312,2010

SILVA, D. K.; NAHAS, M.V. 2004. Atividade física habitual e qualidade de vida relacionada à saúde em mulheres com doença vascular periférica. Revista Brasileira de Ciência e Movimento, Brasília, v.12, n. 4, p. 63-68, 2004.

SILVA, M. F. et al. Relação entre os níveis de atividade física e qualidade de vida de idosos sedentários e fisicamente ativos. Revista Brasileira de Geriatria e Gerontologia, v. 15, n. 4, p. 635-642, 2012.

SILVA, R. S. et al. Atividade física e qualidade de vida. Revista Ciência e Saúde Coletiva, v.15, n.1, p.115-120, 2010.

STEIN, A. M. et al. Nível de atividade física, sono e qualidade de vida de pacientes com doença de Alzheimer.
Revista Brasileira de Atividade Física e Saúde, v.17, n. 3, p. 200-205, 2012.

TAKANO, N. A. et al. Qualidade de vida de idosos com tontura. Revista Brasileira de Otorrinolaringologia, v.76, n. 6, p.769-775, 2010.

TOSCANO, J. J. O.; OLIVEIRA, A. C. C. Qualidade de vida em idosos com distintos níveis de atividade física.

Revista Brasileira de Medicina do Esporte, v.15, n. 3, p.169-173, 2009.

TRIBESS, S.; VIRTUOSO JÚNIOR, J. S.; OLIVEIRA, R. J. Atividade física como preditor da ausência de fragilidade em idosos. Revista da Associação Médica Brasileira, v. 58, n. 3, p. 341-347, 2012.

VECCHIA, R. D. et al. Qualidade de vida na terceira idade, um conceito subjetivo. Revista Brasileira de Epidemiologia, v. 8, n. 3, p. 246-252, 2005.

VITORINO, L. M.; PASKULIN, L. M. G.; VIANNA, L. A. C. Qualidade de vida de idosos em instituição de longa permanência. Revista Latino-Americana de Enfermagem, v. 20, n. 6, p.09, 2012. 